\title{
PIG3 plays an oncogenic role in papillary thyroid cancer by activating the PI3K/AKT/PTEN pathway
}

\author{
JINYE XU ${ }^{1,2}$, JUNBO CAI $^{3}$, XIAOYAN JIN ${ }^{3}$, JINGJIN YANG $^{2}$, QINYAN SHEN $^{1,2}$, \\ XIAOFEI DING ${ }^{2}$ and YONG LIANG ${ }^{1,3}$ \\ ${ }^{1}$ Department of Oncologic Surgery, The First Affiliated Hospital, Wenzhou Medical University, Wenzhou, \\ Zhejiang 325000; ${ }^{2}$ Department of Clinical Laboratory, Medical School, Taizhou University, Taizhou; \\ ${ }^{3}$ Department of Oncologic Surgery, The Affiliated Municipal Hospital, Taizhou University, \\ Taizhou, Zhejiang 318000, P.R. China
}

Received March 23, 2015; Accepted May 18, 2015

DOI: $10.3892 /$ or.2015.4096

\begin{abstract}
The p53-inducible gene 3 (PIG3 or TP53I3) is a downstream gene of $\mathrm{p} 53$, which can be involved in the process of apoptosis induced by 53 via the production of reactive oxygen species (ROS). However, the functional significance of PIG3 in cancer remains to be determined. This aim of this study was to examine the mRNA and protein expression of PIG3 in papillary thyroid carcinoma (PTC) and normal thyroid tissues, assess the relationship between PIG3 expression and clinicopathological parameters in PTC and examine its role in the proliferation of PTC cell lines. The results showed that PIG3 was aberrantly overexpressed in the majority of specimens of PTC while the expression of $\mathrm{p} 53$ was lower in PTC compared with normal thyroid tissues. Anti-PIG3 immunoreactivity positively correlated with TNM grade. In the PTC cell lines, PIG3 silencing using small interfering RNA (siRNAs) impaired their ability of proliferation and decreased the activity of the PI3K/AKT/PTEN pathway. The results suggested that PIG3 plays an oncogenic role in PTC via the regulation of the PI3K/AKT/PTEN pathway and support the exploration of PIG3 as a novel biomarker for patients with PTC.
\end{abstract}

\section{Introduction}

Thyroid cancer has become one of the most common type of endocrine tumors with a rapid growth of incidence in recent decades (1). According to the estimate of the American National Cancer Institute (NCI), 62,980 new cases of thyroid cancer are anticipated in the United States in 2014. Thyroid cancer often occurs in women, and is the fifth leading cancer

Correspondence to: Dr Yong Liang, Department of Oncologic Surgery, The First Affiliated Hospital, Wenzhou Medical University, Wenzhou, Zhejiang 325000, P.R. China

E-mail: liangytu@aliyun.com

Key words: PIG3, p53, proliferation, PI3K/AKT/PTEN pathway, papillary thyroid carcinoma type in women (2). Histologically, over $80 \%$ of thyroid cancer is papillary thyroid carcinoma (PTC), which derives from thyroid follicular cells (3). As a type of well-differentiated cancer, PTC is considered treatable. However, there are $~ 10 \%$ of patients with poor prognosis for recurrence and/or distant metastasis (4). Accordingly, it is extremely essential to clarify the mechanisms of PTC to provide evidence to identify latent biomarkers for early diagnosis, prognosis and therapies.

As a primary member of the quinone oxidoreductase (QOR) family, the p53-inducible gene 3 (PIG3 or TP53I3) was initially identified through the analysis of p53 downstream genes associated with the onset of apoptosis in human colorectal cancer cell (5). PIG3 can be transactivated by p53 with a p53-response element, i.e., a polymorphic microsatellite (TGYCC)n ( $\mathrm{Y}=\mathrm{C}$ or $\mathrm{T}$ ) at its promoter, and the activation degree of PIG3 was determined by the number of repeats of the microsatellite (6). PIG3 is homologous with NADH quinine oxidoreductase 1 (NQO1), and shows oxidoreductase enzymatic activity, which contributes to the process of apoptosis induced by p53 through production of the reactive oxygen species (ROS) (5,7). In addition, PIG3 is a molecule involved in the DNA damage response (DDR) pathway by increasing the phosphorylation of checkpoint kinases including Chk1 and Chk2, and contributing to the recruitment of other DNA repair components (8).

Although the relationship between PIG3 and various types of cancer has been previously investigated, the role of PIG3 in cancer remains to be clarified. As one of the downstream effectors of the important tumor suppressor p53, PIG3 alone is insufficient to induce apoptosis unless activated and cooperating with a set of simultaneously activated pro-apoptotic genes induced by ROS (5). Accumulating evidence has identified the number of repeats of the microsatellite to be correlated with the generation of several types of tumors, although no relationship with increased risk of breast and lung carcinomas was found (9-11). The aforementioned results raised the question regarding the reasons for PIG3 rarely being affected in cancer (12). Recent findings have demonstrated that PIG3 playd a significant role in cancer cell survival. The proliferation ability of PIG3-depleted HeLa cells was found to be decreased despite exhibiting a prolonged progression of G2-M phases (13). In addition, PIG3 participates in the malignant 
development of the disease by creating a connection between oxidative stress and DDR. In $\mathrm{p} 53^{+/+}$cancer cells, intense DNA damage induced by genotoxic/oxidative stress was capable of recruiting a large part of total PIG3 in the nucleus, but failed to activate ROS-dependent apoptosis when other pro-apoptotic genes were not expressed under identical conditions $(8,14)$. This would result in sublethal levels of ROS which continuously maintain the oxidative stress (15). Thus, the continuous demand for PIG3 as a DDR component remains a positive feedback between PIG3 and DNA damage, as the accumulation of DNA damage may lead to mutagenesis, including p53 loss or mutation, which is crucial in carcinogenesis $(16,17)$.

To the best of our best knowledge, however, the clinical and functional significance of PIG3 in PTC remains to be clarified. In this study, we first detected the expression of PIG3 in PTC and normal thyroid tissues and analyzed its clinical significance in PTC. Furthermore, we determined its functional role in PTC cell proliferation and the role of phosphatidylinositide 3-kinases/protein kinase B/phosphatase and tensin homologue deleted on chromosome 10 (PI3K/AKT/PTEN) signaling pathway in a series of assays in vitro. The results showed that PIG3 was aberrantly overexpressed and plays an oncogenic role in PTC.

\section{Materials and methods}

Patients and samples. Consent was provided by all the patients who participated in the study. The study was approved by the Institutional Review Board of the Taizhou Municipal Hospital. In total, 16 male and 54 female patients with PTC (age range, 19-70 years) were recruited for this study and underwent surgery at the Taizhou Municipal Hospital (Zhejian, China) between February and December, 2013. Tumors and normal thyroid tissues were obtained during surgery and immediately stored in liquid nitrogen prior to quantitative PCR and western blot analysis. Matched paraffin-embedded samples used for immunohistochemistry were kindly donated by the Department of Pathology. All the cases were confirmed pathologically and staged on the basis of TNM classification system.

Immunohistochemistry. Immunohistochemical (IHC) staining was used to detect the distribution of PIG3 protein in specimens of PTC and normal thyroid tissue. The excised samples were formalin-fixed, paraffin-embedded and blocks were sectioned serially at $4 \mu \mathrm{m}$ prior to being examined under a microscope. The slides were deparaffinized and rehydrated with xylene in a graded series of ethanol/water concentrations $(100,100,95,90,85$ and $75 \%)$, respectively. Antigen retrieval was achieved by immersing the slides in citrate buffer $(\mathrm{pH}$ 6.0). The slides were autoclaved at $120^{\circ} \mathrm{C}$ for 2 min and then cooled to room temperature. Endogenous peroxidase activity was blocked with $3 \%$ hydrogen peroxide for $10 \mathrm{~min}$ at room temperature. After washing with phosphate-buffered saline (PBS), the samples were treated with 5\% goat serum (ZDR5118; Zhongshan Golden Bridge Biotechnology Co., Ltd., China) to block non-special protein binding for $30 \mathrm{~min}$. The slides were then incubated overnight with primary rabbit polyclonal anti-PIG3 antibody (TA308561; OriGene, Rockville, $\mathrm{MD}, \mathrm{USA}$ ) at a dilution of $1: 800$ at $4^{\circ} \mathrm{C}$ in a humid chamber.
Negative controls (NC) were processed by substituting PBS. After incubation at $37^{\circ} \mathrm{C}$ for $45 \mathrm{~min}$, the sections were rinsed with PBS and incubated with biotinylated goat anti-Rb IgG/ HRP for $1.5 \mathrm{~h}$ at $37^{\circ} \mathrm{C}$. After washing with PBS, the slides were visualized by 3,3'-diaminobenzidine tetrahydrochloride solution (DAB kit, ZLI-9017; Zhongshan Golden Bridge Biotechnology) and counterstained by Mayer's hematoxylin. After being rinsed with water for $20 \mathrm{~min}$, the sections were dehydrated with gradient ethanol sequentially $(75,85,90$, 95,100 and $100 \%$ ) and cleared with xylene. The samples were examined under a microscope (Olympus, Japan) by pathologists (Z.H.Y and L.H.S) who were blinded to the clinicopathological data. The semi-quantitative scoring system used to estimate the expression of PIG3 was combined with staining intensity and the proportion of positive cells (18). The intensity score was graded as 0 (no staining), 1 (weak), 2 (moderate) and 3 (strong). Then, 2,500 cells in five randomly selected areas (magnification, $\mathrm{x} 400$ ) were counted to evaluate the proportion score: the percentage of positive cells $<5,5-35$, $36-70$ and $>70 \%$ were assigned as $0,1,2$ and 3 points, respectively. Multiplication of the intensity and proportion scores was employed to determine the final score. Slides with a score $\leq 3$ were defined as low expression and those with a score $\geq 4$ were regarded as high expression.

Cell culture. Cell culture reagents were obtained from Gibco (Carlsbad, CA, USA). Human PTC CGTHW-3 and K1 cells were purchased from the American Type Culture Collection (ATCC; Manassas, VA, USA). The two cell lines were cultured in RPMI-1640, supplemented with $10 \%$ fetal bovine serum (FBS) in a modulator incubator chamber and maintained at $37^{\circ} \mathrm{C}$ and $5 \% \mathrm{CO}_{2}$.

Cell transfection. The RNAi-Mate for cell transfection, small interfering RNA (siRNA) for human PIG3 (sense, 5'-AAAUG UUCAGGCUGGAGACUAdTdT-3' and antisense, 5'-UAGUC UCCAGCCUGAACAUUUdTdT-3') and its corresponding NC (sense, 5'-CCUACGCCAAUUUCGUdTdT-3' and antisense, 5'-ACGAAAUUGGUGGCGUAGGdTdT-3') were purchased from Shanghai GenePharma Co., Ltd. (Shanghai, China). When the GTHW-3 and K1 cells grew to 40-60\% confluence in $24 \mathrm{~h}$, the medium was removed from each plate and siRNA/RNAi-Mate complexes incubated in serum-free Opti-MEM 1 (Gibco) were added to the cells according to the manufacturer's instructions. The final concentration of siRNA in each plate was $20 \mathrm{nM}$. After 6-8 h, the medium was replaced with RPMI-1640 containing 10\% FBS. After being cultured for an additional 48 or $72 \mathrm{~h}$, the cells were harvested for quantitative PCR and western blot analysis, respectively.

Cell viability assay. Cells (5,000 CGTHW-3 or 6,500 K1) were seeded in 96-well plates/well and cultured overnight. The two types of cells were transfected with PIG3 siRNA and NC respectively, and each experiment was performed in triplicate. After $48 \mathrm{~h}$ of transfection, each well was treated with $10 \mu \mathrm{l}$ CCK-8 (Beyotime, Jiangsu, China) solution. The cells were incubated in the modulator incubator chamber for another $2 \mathrm{~h}$. The optical density (OD) of each well was determined at $450 \mathrm{~nm}$ using a 550 Microplate Reader (Bio-Rad, Hercules, CA, USA). 
Table I. Primer sequences used for quantitative PCR.

\begin{tabular}{lcc}
\hline Genes & \multicolumn{1}{c}{ Forward } & Reverse \\
\hline PIG3 & 5'-AGCCGGGCCAGGAGTAAGTAAC-3' & 5'-GCCGAAGAGGATCAGGCAAAT- 3' \\
p53 & 5'-GGC CCA CTT CAC CGT ACT AA-3' & 5'-GTG GTT CTT TCA AGG CCAGATGT-3' \\
GAPDH & 5'-CATCAGCAA TGCCTCCTGCAC-3' & 5'-TGAGTCCTTCCACGATACCAA AGTT-3' \\
\hline
\end{tabular}

Colony formation assay. The CGTHW-3 and K1 cells were transfected with PIG3 siRNA or NC as previously described. After $48 \mathrm{~h}$, the transfected cells were seeded in 6-well plates at a density of 800 cells/well, and allowed to form colonies for 10 days with completed medium. The colonies were fixed in $10 \%$ methanol for 15 min and stained with crystal violet for $25 \mathrm{~min}$ at room temperature. The number of colonies with $>50$ cells were counted manually. Each experiment was conducted in triplicate three times.

Quantitative PCR ( $q P C R$ ). Total RNA was extracted from thyroid tissues and cells using TRIzol reagent (Invitrogen-Life Technologies, Carlsbad, CA, USA). The concentration of total RNA was determined by a UV spectrophotometer, and all the isolated RNA samples had an A260/A280 nm ratio of $>1.8$. Total RNA was reverse transcribed using a cDNA Reverse Transcription kit (Tiangen Biotechnology, Beijing, China). As per the manufacturer's instructions, the final reaction volume was $20 \mu \mathrm{l}$. Then, $2.0 \mu \mathrm{l} \mathrm{cDNA}$ was added to the qPCR reaction system of a total volume of $20 \mu$ l, which contained a $9 \mu \mathrm{l}$ mixture of 2.5X Real Master mix/20X SYBR solution, $0.5 \mu 1$

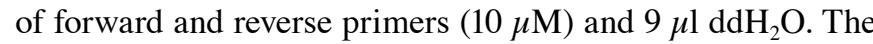
primers used in the qPCR assays to express the investigated transcripts were produced at Invitrogen-Life Technologies (Table I). GAPDH served as the reference gene for analysis. The qPCR assays were run in triplicate on the ABI 7300 Real Time PCR system (Applied Biosystems Life Technologies, Foster City, CA, USA) under the following cycling conditions: $95^{\circ} \mathrm{C}$ for $2 \mathrm{~min}, 40$ cycles of $95^{\circ} \mathrm{C}$ for $15 \mathrm{sec}, 58^{\circ} \mathrm{C}$ for $30 \mathrm{sec}$ and $68^{\circ} \mathrm{C}$ for $60 \mathrm{sec}$. The comparative $\mathrm{Ct}$ method was used to calculate the relative expression of mRNA level.

Western blot analysis. Rabbit antibodies of anti-PI3K, anti-AKT, anti-P-AKT, anti-P53, anti-PTEN and anti-GAPDH were purchased from Cell Signaling Technology (Beverly, MA, USA) and anti-PIG3 was purchased from OriGene. The total protein of each sample was extracted using T-PER solution (Thermo Fisher Scientific, Waltham, MA, USA) according to the manufacturer's instructions and then quantified with BSA standard methodology. An equivalent amount of protein was loaded and fractionated by SDS-polyacrylamide gel electrophoresis, and electrotransferred onto PVDF membranes. After blocking with 5\% non-fat milk, the membranes were blotted with each of primary antibodies (1:1,000 dilution) overnight at $4^{\circ} \mathrm{C}$. The membranes were treated with a horseradish peroxidase-conjugated secondary antibody at a dilution of 1:10,000 for $1.5 \mathrm{~h}$ at room temperature. The blots were visualized using ECL Plus Western Blotting Detection reagents (Beyotime) and scanned in ImageQuant LAS 4000 Mini (GE Healthcare, Pittsburgh, PA, USA).
Table II. Expression of PIG3 in PTC and in normal thyroid tissue.

\begin{tabular}{lcccc}
\hline Groups & Positive & Negative & $\chi^{2}$ & P-value \\
\hline PTC tissue & 52 & 18 & & \\
Normal thyroid tissue & 21 & 34 & 16.525 & $0.000^{\mathrm{a}}$ \\
\hline
\end{tabular}

${ }^{\mathrm{a}} \mathrm{P}<0.05 ;$ PTC, papillary thyroid carcinoma.

Statistical analysis. Experiments were conducted independently three times. SPSS 17.0 software was used for the statistical analysis. The differences in the IHC staining in thyroid tissues and the relationship between protein expression levels and clinicopathological characteristics were calculated using the $\chi^{2}$ test. The qPCR data, western blot data, colony formation and CCK-8 data were recorded as numeric data and presentedas the mean \pm standard deviation (SD). The Student's t-test and one-way ANOVA analysis were used to compare the means between 2-3 groups, respectively. $\mathrm{P}<0.05$ was regarded as statistically significant.

\section{Results}

PIG3 is highly expressed in PTC tissues. IHC was performed to detect the expression of PIG3 in PTC and normal thyroid tissues. The PIG3 protein foci were distributed on the nuclear and cytoplasm in PTC and exhibited a higher expression with total immunoreactive positive rate of $74 \%$ (52/70) (Fig. 1B-B'), which was significantly higher than the $32 \%(21 / 65)$ in normal thyroid tissues (Fig. 1A-A'; P $<0.001$; Table II). qPCR and western blotting confirmed that PIG3 expression was consistent with the IHC results at the mRNA and protein level, respectively, in PTC (Fig. 2; P<0.01).

The relationship between PIG3 expression and the clinicopathologic parameters of the 70 cases of PTC were assessed. The results indicated that PIG3 expression was positively associated with TNM grade (Table III; $\mathrm{P}<0.05$ ), while no association between PIG3 expression and age, gender, tumor size and lymphatic metastasis was identified (Table III; $\mathrm{P}>0.05$ ).

PIG3 has been proven to be one of the downstream effectors of the important tumor suppressor p53. To investigate whether the high expression of PIG3 in PTC was induced by p53, we determined the expression of p53 in PTC and normal thyroid tissues by qPCR and western blotting. In contrast to PIG3, the expression of p53 in PTC was lower than normal thyroid tissues at the mRNA and protein level (Fig. 2; $\mathrm{P}<0.01$ ). 

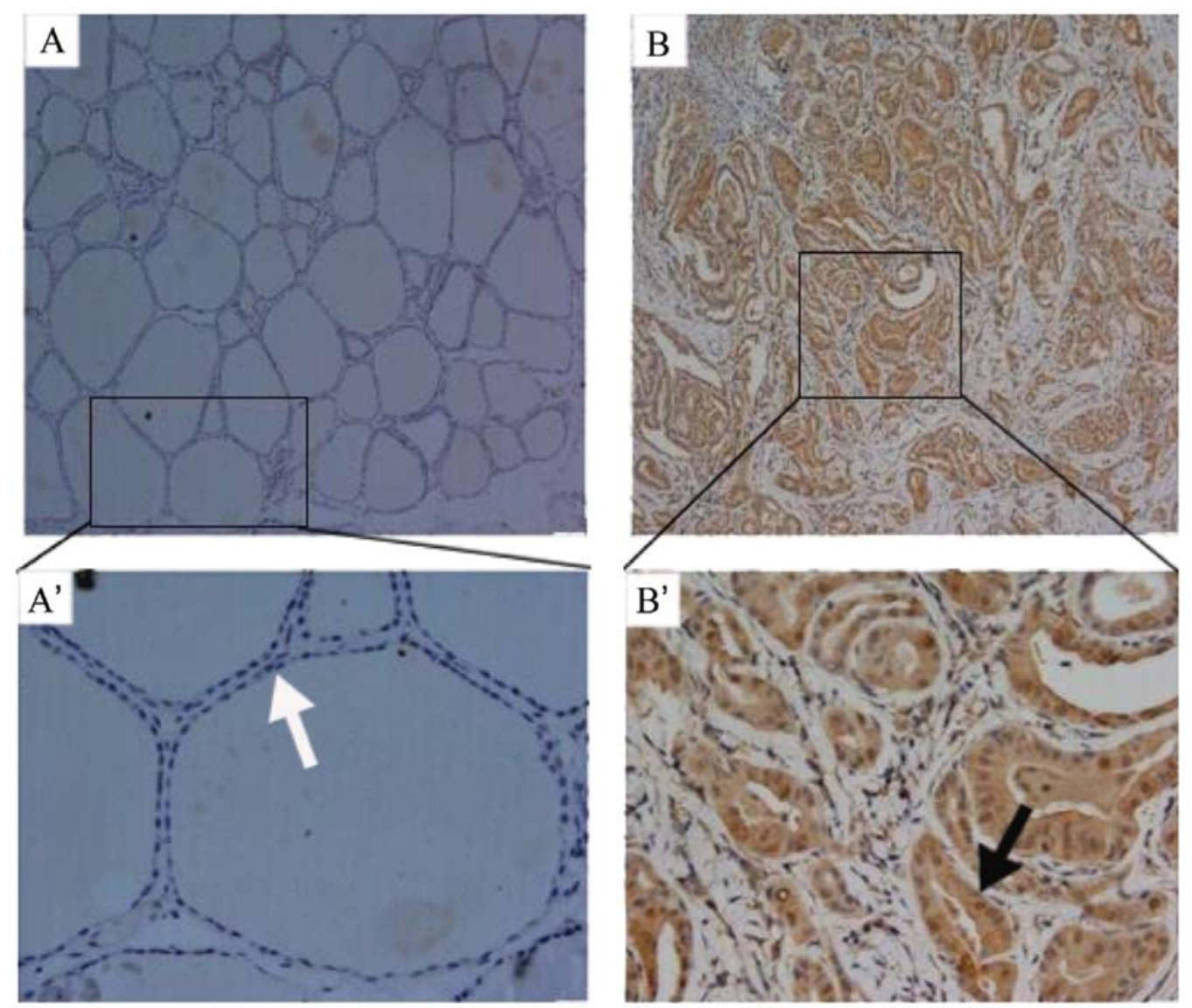

Figure 1. Expression of PIG3 in PTC and normal thyroid tissues determined by immunohistochemistry. The PIG3 expression in normal follicular cells is noted by the white arrow (A,A'). PTC showing strong PIG3 expression (black arrow) (B, B') (Magnification, x100 and 400 for A and B, and A' and B'). PTC, papillary thyroid carcinoma.

Table III. Relationship between PIG3 protein expression and clinicopathological characteristics of PTC.

\begin{tabular}{lccccc}
\hline Variables & No. & Positive & Negative & $\chi^{2}$ & P-value \\
\hline Gender & & & & & \\
$\quad$ Male & 16 & 11 & 5 & 0.063 & 0.802 \\
$\quad$ Female & 54 & 41 & 13 & & \\
Age (years) & & & & & \\
$\quad<45$ & 39 & 27 & 12 & 1.178 & 0.278 \\
$\geq 45$ & 31 & 25 & 6 & & \\
Tumor size (cm) & & & & & \\
$\leq 2$ & 54 & 38 & 16 & 1.105 & 0.293 \\
$>2$ & 16 & 14 & 2 & & \\
Lymphatic & & & & & \\
metastasis & & & & & \\
Yes & 46 & 35 & 11 & 0.228 & 0.633 \\
No & 24 & 17 & 7 & & \\
TNM stages & & & & & \\
I+II & 49 & 33 & 16 & 4.117 & $0.042^{\mathrm{a}}$ \\
III+IV & 21 & 19 & 2 & & \\
\hline
\end{tabular}

${ }^{\mathrm{a}} \mathrm{P}<0.05 ; \mathrm{PTC}$, papillary thyroid carcinoma.
Silencing PIG3 induces anti-proliferative effect in PTC cell lines. To determine whether the downregulation of PIG3 affected the biological behavior of PTC cell lines, PIG3 siRNA and the corresponding NC were transfected into CGTHW-3 and $\mathrm{K} 1$ cells. The two types of cells showed a significant decrease in PIG3 mRNA and protein expression levels in the group of siRNA, compared with the untreated group (MOCK) and the NC group (Fig. 3A-C; $\mathrm{P}<0.05$ ).

CCK-8 assay was used to examine the effect of silencing PIG3 on the proliferation of PTC cells. Silencing PIG3 significantly decreased the viability of CGTHW-3 and K1 cells at $48 \mathrm{~h}$ after transfection, compared with the NC group (Fig. 3D, $\mathrm{P}<0.05)$. The colony formation assay also showed the effects of PIG3 knockdown on the growth of PTC cells. Compared with the NC group, the downregulation of PIG3 suppressed the colony formation ability of the two PTC cell types (Fig. 3E and $\mathrm{F}, \mathrm{P}<0.05)$. These data suggested that PIG3 played an important role in promoting the proliferation of PTC cells.

Knockdown of PIG3 suppresses activation of the PI3K/AKT signaling pathway. PI3K/AKT/PTEN is a classical signaling pathway involved in many cancer types which enhance malignant cell proliferation. To identify the exact mechanisms involved in PIG3 promotion of PTC cell proliferation. Activation of the PI3K/AKT/PTEN signaling pathway was determined by using western blot analysis after silencing the 
A

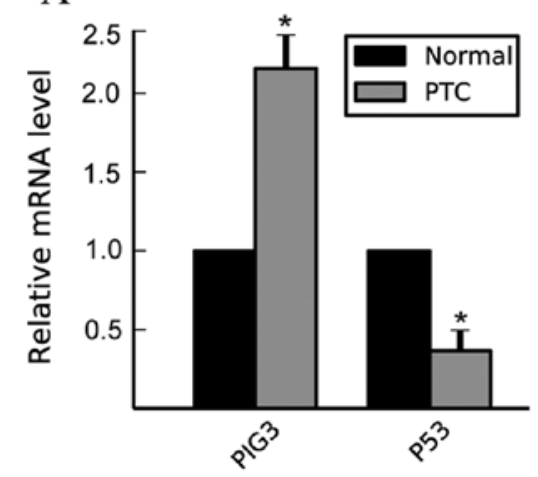

B

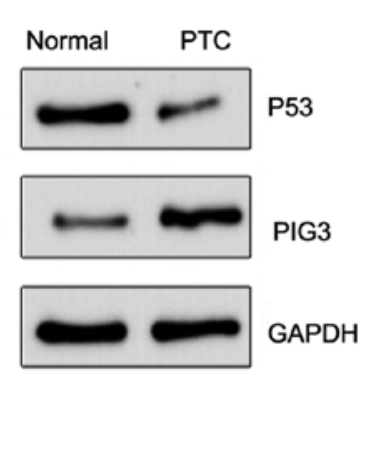

C

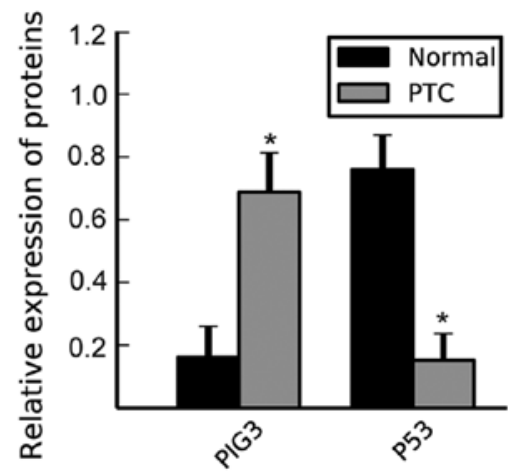

Figure 2. Expression of PIG3 and P53 in PTC ( $\left.{ }^{*} \mathrm{P}<0.05\right)$. (A) The relative mRNA levels of PIG3 and p53 in normal thyroid tissue and PTC were determined by qPCR, with GAPDH as a control. (B and C) PIG3 and p53 protein expression was detected in PTC and normal thyroid tissue using western blot analysis. Each bar represents the mean of three independent.

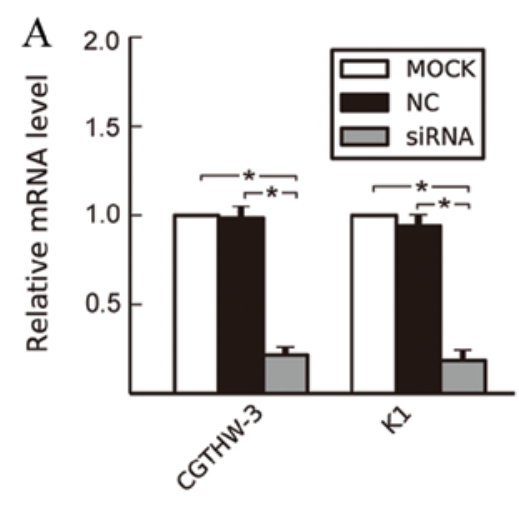

D

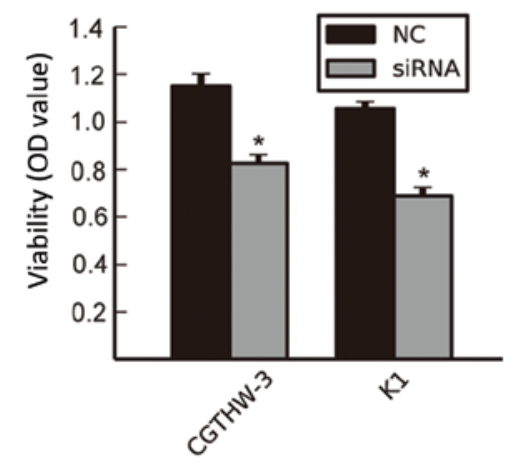

B

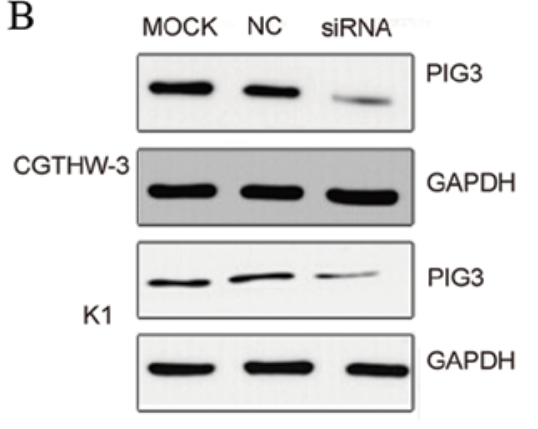

E

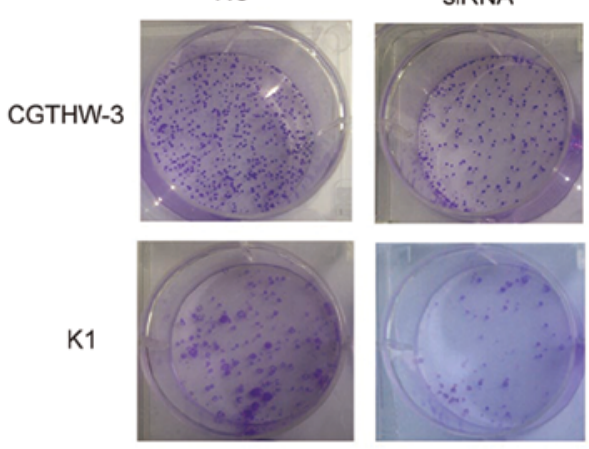

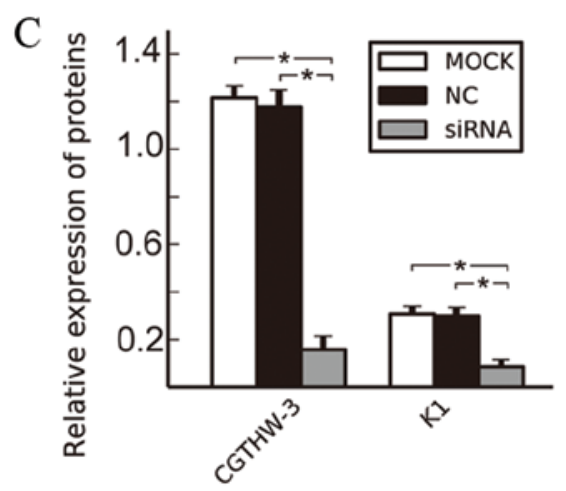

F

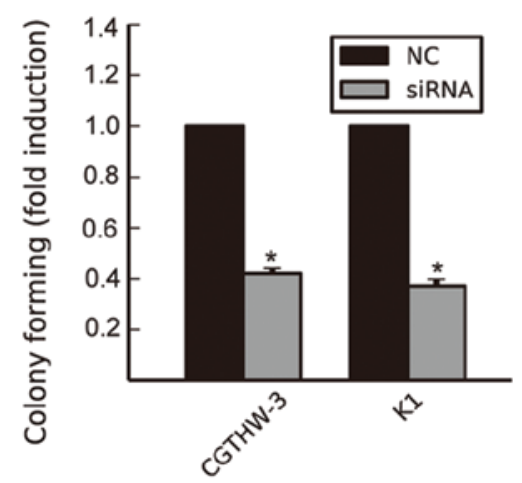

Figure 3. Downregulation of PIG3 in PTC cells and effects of PTC on the colony formation and cell proliferation of CGTHW-3 and K1 cells ( $\mathrm{P}<0.05$ ). (A) PIG3 mRNA levels were analyzed by qPCR. PTC cells were transfected with siRNA for PIG3 (siRNA) and NC, the untreated group (MOCK) with GAPDH as a control. (B and C) PIG3 protein levels in the three groups were analyzed using western blot analysis. GAPDH was used as an internal control. (D) CCK-8 cell proliferation assay for siRNA and NC-transfected PTC cells. (E and F) Representative colony formation assay, the numbers of colonies in NC were set as 1 . Each bar shows the mean of three independent experiments. PTC, papillary thyroid carcinoma; NC, negative control.

expression of PIG3 in CGTHW-3 and K1 cells. The protein expression levels of PTEN were markedly increased while PI3K p110a was significantly reduced (Fig. 4; P<0.05) and the expression of AKT exhibited no notable difference between the siRNA and NC groups. By contrast, the amount of p-AKT markedly decreased in cells transfected with siRNA (Fig. 4, $\mathrm{P}<0.05)$. These data suggested that the downregulation of PIG3 suppressed the proliferation of PTC cells via regulation of the PI3K/AKT/PTEN signaling pathway.

\section{Discussion}

PIG3 was initially isolated through analysis of the p53 downstream genes associated with the onset of apoptosis in 1997 (5). Although several studies have identified an association between PIG3 and human cancers, the role of PIG3 in cancers remains to be determined. In the present study, we showed that the expression of PIG3 in PTC was significantly higher than that in normal thyroid tissues at the mRNA and 

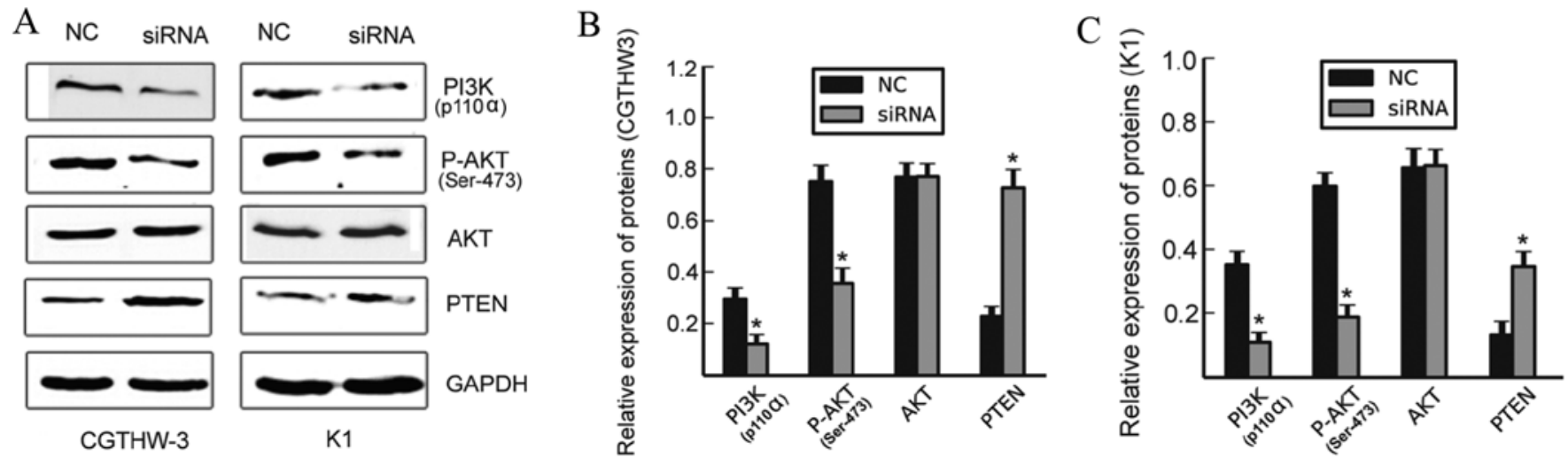

Figure 4. Knockdown of PIG3 modulation of PI3K/AKT/PTEN pathway activity in PTC cells ( $\left.{ }^{*}<0.05\right)$. (A and C) PI3K p110a, AKT, P-AKT and PTEN protein levels in the 2 groups were analyzed by western blot analysis after transfection with siRNA or NC in CGTHW-3 and K1 cells. Each bar represents the mean value and error information. $\mathrm{NC}$, negative control.

protein level. We assessed the relationship between PIG3 expression and clinicopathological parameters of the 70 cases of PTC. The results indicated a positive association of PIG3 expression with TNM grade. Since PIG3 can be mediated by p53 mainly through a microsatellite (TGYCC)n ( $\mathrm{Y}=\mathrm{C}$ or $\mathrm{T})$ at its promoter and the extent of PIG3 activation was determined by the number of repeats of the microsatellite (6). The expression of p53 was also detected in these thyroid tissues. Notably, the expression of p53 was lower in PTC compared with that in the normal thyroid tissues. We also demonstrated that PIG3 promoted PTC cell proliferation by modulating the PI3K/AKT/PTEN signing pathway. Our results suggest that PIG3 plays an essential role in facilitating the proliferation of PTC and that the expression of PIG3 is not associated with p53 in PTC.

PIG3 has been proven to be one of the downstream effectors of the important tumor suppressor p53 and may be induced by 553 under cell stress and control conditions (19). When cooperated with other p53 downstream pro-oxidative genes, PIG3 participated in the process of apoptosis induced by p53 by producing ROS (5). However, the expression of PIG3 was not only induced by p53. Previous findings have demonstrated that the variable number of tandem repeats (VNTRs) of pentanucleotides (TGYCC)n at the promoter of PIG3 was reported to be correlated with the generation of squamous cell carcinoma of the head and neck and invasive bladder cancer $(10,11)$. In addition, after p53 stimulus was removed from cells, elevated levels of PIG3 were maintained while p53, MDM2 and p21 protein levels decreased rapidly (19). The present study focused on determining the relationship between PIG3 and p53 in PTC and found that PIG3 can be induced by molecules other than p53, which may identify the molecular mechanisms involved in PIG3 contribution to carcinogenesis and development in human cancers.

Sustained proliferation is one of the most fundamental features of cancer cells (20). Our findings show that silencing PIG3 significantly decreased the viability and colony formation ability of CGTHW-3 and K1 cells, which is similar to previously obtained results $(13,19)$. A recent finding showed that the proliferation capability of HeLa cells decreased when PIG3 was knocked down despite elongating the G2-M phase (13). Although PIG3 levels were elevated during p53-mediated growth arrest, the expression of PIG3 remained relatively constant in cells that resumed cycling when the expression of p53 was decreased (19). Additionally, the ROS level in cells and their condition is a crucial regulatory factor that directly determines the fate of the cells, i.e., proliferation, apoptosis and migration (21). Under some conditions when the selective expression of pro-apoptotic genes was induced by p53, PIG3 could produce sublethal levels of ROS, but failed to activate ROS-dependent apoptosis, thus playing a vital role in carcinogenesis (22). The results indicate that PIG3 potentially plays an oncogenic role in human cancers.

We determined the exact mechanisms of how PIG3 promoted the proliferation of PTC cells. The activity of the $\mathrm{PI} 3 \mathrm{~K} / \mathrm{AKT} / \mathrm{PTEN}$ signaling pathway was also determined by using siRNA to silence PIG3 expression. The PI3K/AKT/PTEN signing pathway plays a pivotal role in cell proliferation, and survival in human cancers including those deriving in the thyroid gland $(23,24)$. Previous findings have demonstrated that ROS increases the expression of PI3K and inactivates PTEN directly, and can mediate cell survival and proliferation by modulating the PI3K/AKT/PTEN pathway $(25,26)$. However, the relationship between PIG3 and the PI3K/AKT/PTEN signaling pathway remains unknown. In the present study, we found that after knockdown of PIG3 in PTC cells, the PI3K/ AKT pathway was inactivated while the expression of PTEN was markedly increased. PIG3 silencing suppresses the activation of PI3K/AKT/PTEN signaling pathway. Our results and those of previous studies suggest that PIG3 probably regulates the expression of the PI3K/AKT/PTEN signaling pathway by producing ROS in PTC. However, a detailed elucidation of the connection between these molecules remains to be determined in future studies.

In summary, our study has demonstrated that PIG3 is highly expressed in PTC and may promote the proliferation of PTC via regulation of the PI3K/AKT/PTEN pathway, which indicates that PIG3 possibly plays an oncogenic role in PTC and may serve as a new target for the clinical diagnosis and treatment of PTC. Moreover, we found that the expression of PIG3 in PTC was not induced by p53. However, the molecular basis of how PIG3 is activated and how PIG3 regulates the expression of the PI3K/AKT/PTEN signaling pathway in PTC remain important issues to be investigated. 


\section{Acknowledgements}

We would like to thank Professor David T. Yew (Department of Anatomy Chinese University of Hong Kong) for carefully editing the manuscript and sincerely acknowledge Dr Huayuan Zhan (Department of Pathology, The Affiliated Municipal Hospital) for the technical support received for the frozen sections. This study was supported by the National Natural Science Foundation of China (no. 81072209).

\section{References}

1. Kweon KH, Lee CR, Jung SJ, Ban EJ, Kang SW, Jeong JJ, Nam KH, Jo YS, Lee J and Chung WY: Sirt1 induction confers resistance to etoposide-induced genotoxic apoptosis in thyroid cancers. Int J Oncol 45: 2065-2075, 2014.

2. Xing Y, Luo DY, Long MY,Zeng SL and Li HH: High ALDH1A1 expression correlates with poor survival in papillary thyroid carcinoma. World J Surg Oncol 12: 29, 2014.

3. Tang C, Yang L, Wang N, Li L, Xu M, Chen GG and Liu ZM: High expression of GPER1, EGFR and CXCR1 is associated with lymph node metastasis in papillary thyroid carcinoma. Int J Clin Exp Pathol 7: 3213-3223, 2014.

4. Minna E, Romeo P, De Cecco L, Dugo M, Cassinelli G, Pilotti S, Degl'Innocenti D, Lanzi C, Casalini P, Pierotti MA, et al: miR-199a-3p displays tumor suppressor functions in papillary thyroid carcinoma. Oncotarget 5: 2513-2528, 2014.

5. Polyak K, Xia Y, Zweier JL, Kinzler KW and Vogelstein B: A model for p53-induced apoptosis. Nature 389: 300-305, 1997.

6. Contente A, Dittmer A, Koch MC, Roth J and Dobbelstein M: A polymorphic microsatellite that mediates induction of PIG3 by p53. Nat Genet 30: 315-320, 2002.

7. Porté S, Valencia E, Yakovtseva EA, Borràs E, Shafqat N, Debreczeny JE, Pike AC, Oppermann U, Farrés J, Fita I, et al: Three-dimensional structure and enzymatic function of proapoptotic human p53-inducible quinone oxidoreductase PIG3. J Biol Chem 284: 17194-17205, 2009.

8. Lee JH, Kang Y, Khare V, Jin ZY, Kang MY, Yoon Y, Hyun JW, Chung MH, Cho SI, Jun JY, et al: The p53-inducible gene 3 (PIG3) contributes to early cellular response to DNA damage. Oncogene 29: 1431-1450, 2010

9. Gorgoulis VG, Liloglou T, Sigala F, Korkolis D, Yannoukakos D, Papalambros E, Asimacopoulos PJ, Papavassiliou AG and Kotsinas A: Absence of association with cancer risk and low frequency of alterations at a p53 responsive PIG3 gene polymorphism in breast and lung carcinomas. Mutat Res 556 143-150, 2004.

10. Ito M, Nishiyama H, Watanabe J, Kawanishi H, Takahashi T, Kamoto T, Habuchi T and Ogawa O: Association of the PIG3 promoter polymorphism with invasive bladder cancer in a Japanese population. Jpn J Clin Oncol 36: 116-120, 2006.

11. Guan X, Liu Z, Wang L, Wang LE, Sturgis EM and Wei Q: Functional repeats (TGYCC)n in the p53-inducible gene 3 (PIG3) promoter and susceptibility to squamous cell carcinoma of the head and neck. Carcinogenesis 34: 812-817, 2013.
12. Kotsinas A,Pateras IS, Galanos PS, Karamouzis MV, Sfikakis PP and Gorgoulis VG: Why is p53-inducible gene 3 rarely affected in cancer? Oncogene 29: 5220, 2010.

13. Li B, Shang ZF, Yin JJ, Xu QZ, Liu XD, Wang Y, Zhang SM, Guan $\mathrm{H}$ and Zhou PK: PIG3 functions in DNA damage response through regulating DNA-PKcs homeostasis. Int J Biol Sci 9: 425-434, 2013.

14. Xie P, Tian C, An L, Nie J, Lu K, Xing G, Zhang L and He F: Histone methyltransferase protein SETD2 interacts with p53 and selectively regulates its downstream genes. Cell Signal 20: 1671-1678, 2008.

15. Kryston TB, Georgiev AB, Pissis P and Georgakilas AG: Role of oxidative stress and DNA damage in human carcinogenesis. Mutat Res 711: 193-201, 2011.

16. Moreli JB, Santos JH, Rocha CR, Damasceno DC, Morceli G, Rudge MV, Bevilacqua E and Calderon IM: DNA damage and its cellular response in mother and fetus exposed to hyperglycemic environment. Biomed Res Int 2014: 676758, 2014.

17. Halazonetis TD, Gorgoulis VG and Bartek J: An oncogene-induced DNA damage model for cancer development. Science 319: 1352-1355, 2008.

18. Chambers JT, Carcangiu ML, Voynick IM and Schwartz PE: Immunohistochemical evaluation of estrogen and progesterone receptor content in 183 patients with endometrial carcinoma. Part II: Correlation between biochemical and immunohistochemical methods and survival. Am J Clin Pathol 94: 255-260, 1990.

19. Flatt PM, Polyak K, Tang LJ, Scatena CD, Westfall MD, Rubinstein LA, Yu J, Kinzler KW, Vogelstein B, Hill DE, et al: p53-dependent expression of PIG3 during proliferation, genotoxic stress, and reversible growth arrest. Cancer Lett 156: 63-72, 2000.

20. Hanahan D and Weinberg RA: Hallmarks of cancer: The next generation. Cell 144: 646-674, 2011.

21. Ivanova D, Bakalova R, Lazarova D, Gadjeva V and Zhelev Z: The impact of reactive oxygen species on anticancer therapeutic strategies. Adv Clin Exp Med 22: 899-908, 2013.

22. Kotsinas A, Aggarwal V, Tan EJ, Levy B and Gorgoulis VG: PIG3: A novel link between oxidative stress and DNA damage response in cancer. Cancer Lett 327: 97-102, 2012.

23. Liu R, Liu D, Trink E, Bojdani E, Ning G and Xing M: The Akt-specific inhibitor MK2206 selectively inhibits thyroid cancer cells harboring mutations that can activate the PI3K/Akt pathway. J Clin Endocrinol Metab 96: E577-E585, 2011.

24. Lim HJ, Crowe P and Yang JL: Current clinical regulation of $\mathrm{PI} 3 \mathrm{~K} / \mathrm{PTEN} / \mathrm{Akt} / \mathrm{mTOR}$ signalling in treatment of human cancer. J Cancer Res Clin Oncol 141:671-689, 2015.

25. Korbecki J, Baranowska-Bosiacka I, Gutowska I and Chlubek D: The effect of reactive oxygen species on the synthesis of prostanoids from arachidonic acid. J Physiol Pharmacol 64: 409-421, 2013.

26. Kumar B, Koul S, Khandrika L, Meacham RB and Koul HK: Oxidative stress is inherent in prostate cancer cells and is required for aggressive phenotype. Cancer Res 68: 1777-1785, 2008. 